\title{
Response to mTOR inhibition: activity of elF4E predicts sensitivity in cell lines and acquired changes in elF4E regulation in breast cancer
}

\author{
Sampoorna Satheesha', Victoria J Cookson', Louise J Coleman', Nicola Ingram¹, Brijesh Madhok', \\ Andrew M Hanby ${ }^{2}$, Charlotte AB Suleman², Vicky S Sabine ${ }^{3}$, E Jane Macaskill ${ }^{3}$, John MS Bartlett ${ }^{3,4}$, \\ J Michael Dixon ${ }^{4}$, Jim N McElwaine ${ }^{5}$, Thomas A Hughes ${ }^{1 *}$
}

\begin{abstract}
Background: Inhibitors of the kinase mTOR, such as rapamycin and everolimus, have been used as cancer therapeutics with limited success since some tumours are resistant. Efforts to establish predictive markers to allow selection of patients with tumours likely to respond have centred on determining phosphorylation states of mTOR or its targets 4E-BP1 and S6K in cancer cells. In an alternative approach we estimated elF4E activity, a key effector of mTOR function, and tested the hypothesis that eIF4E activity predicts sensitivity to mTOR inhibition in cell lines and in breast tumours.
\end{abstract}

Results: We found a greater than three fold difference in sensitivity of representative colon, lung and breast cell lines to rapamycin. Using an assay to quantify influences of elF4E on the translational efficiency specified by structured $5^{\prime} U T R$, we showed that this estimate of elF4E activity was a significant predictor of rapamycin sensitivity, with higher elF4E activities indicative of enhanced sensitivity. Surprisingly, non-transformed cell lines were not less sensitive to rapamycin and did not have lower elF4E activities than cancer lines, suggesting the mTOR/4E-BP1/elF4E axis is deregulated in these non-transformed cells. In the context of clinical breast cancers, we estimated elF4E activity by analysing expression of elF4E and its functional regulators within tumour cells and combining these scores to reflect inhibitory and activating influences on elF4E. Estimates of elF4E activity in cancer biopsies taken at diagnosis did not predict sensitivity to 11-14 days of pre-operative everolimus treatment, as assessed by change in tumour cell proliferation from diagnosis to surgical excision. However, higher pre-treatment elF4E activity was significantly associated with dramatic post-treatment changes in expression of elF4E and $4 \mathrm{E}$-binding proteins, suggesting that elF4E is further deregulated in these tumours in response to mTOR inhibition.

Conclusions: Estimates of elF4E activity predict sensitivity to mTOR inhibition in cell lines but breast tumours with high estimated eIF4E activity gain changes in elF4E regulation in order to enhance resistance.

\section{Background}

Rapamycin is an immunosuppressant drug prescribed for prophylaxis of organ rejection following renal transplant [1]. Recently it, and derivatives such as everolimus, have been tested as cancer therapeutics with some success [2-5]. The drugs inhibit the serine/threonine-specific protein kinase mTOR (mammalian Target Of Rapamycin) by forming a complex with another protein, FKBP12 (FK

\footnotetext{
* Correspondence: t.hughes@leeds.ac.uk

'Leeds Institute of Molecular Medicine, St. James's University Hospital, Leeds University, Leeds, LS9 7TF, UK

Full list of author information is available at the end of the article
}

506-binding protein of $12 \mathrm{kDa}$ ), that then associates with mTOR. This association allosterically inhibits mTOR's ability to assemble the functionally active complex mTORC1 (mTOR complex 1) [6,7]. In addition, at high doses the drugs can bind directly to mTOR inhibiting its function [8]. mTORC1 activity is up-regulated in many cancers as a result of loss of function of tumour suppressor genes such as p53 or LKB1, up-regulation of AKT, or mitogenic signalling [9-11]. Pathways downstream of mTORC1 that contribute to carcinogenesis have also been defined. The main mTORC1 targets are the eIF4Ebinding proteins (4E-BP1, 2 and 3) and the S6 protein

\section{Ciomed Central}


kinases (S6K1 and 2) [12,13]. Hypophosphorylated $4 \mathrm{E}-\mathrm{BPs}$ bind to and inhibit the translation factor eIF4E, while these interactions are inhibited by mTORC1dependent 4E-BP phosphorylation, releasing active eIF4E [14]. S6K activity is stimulated by phosphorylation by mTORC1. The result of increased activity of both eIF4E and S6K is changes in translation. Increased eIF4E activity enhances cap-dependent translation of mRNAs with a high degree of secondary structure within their 5' untranslated regions (UTRs) $[15,16]$, a subset of transcripts greatly enriched for cancer-related messages [17]. In addition, nuclear export of some cancer-related transcripts is stimulated by highly active eIF4E $[18,19]$. Increased S6K activity leads to up-regulation of overall translational capacity, as a result of increased ribosome biogenesis, and may also contribute to enhanced translation of transcripts with structured 5'UTRs via upregulation of the activity of the translation factor eIF4A [20]. Therefore, increased mTORC1 activity in cancer enhances expression of key oncogenes and increases cellular growth potential. Reversing these effects, and thereby reducing cell growth or inducing apoptosis, is thought to be the basis of the therapeutic action of mTOR inhibitors in cancer.

However, mTOR inhibitors have proved less successful in cancer clinical trials than might be hoped from the importance of the molecular pathways involved [2]. This relates partly to some toxicity in non-target tissues $[21,22]$, but also to intrinsic or acquired resistance in many individual cancers. Consequently, there is a need for predictive biomarkers to allow selection of patients with cancers most likely to respond to such agents. A number of potential biomarkers have been discussed in the literature, focusing on expression levels or phosphorylation states of mTOR itself [23], or the immediate targets of mTORC1, 4E-BP1 $[24,25]$ and S6K1 $[26,27]$. Here, we take a different approach and estimate the activity of eIF4E, one of the key effectors of mTORC1 function, and investigate whether this reflects response to mTOR inhibition in both tissue culture and in clinical breast cancers.

\section{Methods}

\section{Cell culture, transfection, proliferation assays}

Cell lines were obtained from American Tissue Culture Collection or European Collection of Animal Cell Cultures and were maintained at $37^{\circ} \mathrm{C}$ in humidified air $/ 5 \%$ $\mathrm{CO}_{2}$. Bi-monthly mycoplasma checks (MycoAlert assay; Lonza, Basel, Switzerland) were consistently negative. Cellspecific culture/transfection conditions are described in Additional file 1, Table S1. Plasmids pTH-GFPa (control GFP reporter) [28], GFP+60 (structured GFP reporter) [29] and pcDNA3HA-eIF4E [30] have been described previously. For proliferation assays, cells were plated into 96- well, flat-bottomed plates at $5 \times 10^{3}-2 \times 10^{4}$ cells/well (depending on cell line growth characteristic to ensure continued log phase growth; this was assessed by examination of growth of DMSO-treated cells over $48 \mathrm{~h}$ ). Five replicate wells were treated with DMSO (control) or InSolution $^{\text {TM }}$ Rapamycin (Calbiochem, Darmstadt, Germany) for 24 (data not shown) or $48 \mathrm{~h}$. Metabolically active cells were quantified by assessing conversion of 3-(4,5Dimethyl-2-thiazolyl)-2,5-diphenyl-2H-tetrazolium bromide (Sigma-Aldrich, Dorset, UK) to formazan. Formazan was dissolved in propan-1-ol and quantified as absorbance at $570 \mathrm{~nm}$ (Opsys, Dynex, Sussex, UK).

\section{Western blotting}

Proteins were extracted in RIPA $(50 \mathrm{mM}$ Tris $\mathrm{HCl} \mathrm{pH}$ 7.4, $150 \mathrm{mM} \mathrm{NaCl}, \mathrm{NP} 40$ 1\%, Complete inhibitors [Roche, Basel, Switzerland]) and were quantified in triplicate with the RCDC protein assay (BioRad, Hercules, USA). $20 \mu \mathrm{g}$ of protein was loaded into wells of $12 \%$ NuPAGE bis-tris gels running in MOPS NuPAGE buffer (Invitrogen, Paisley, UK). Proteins were transferred to PDVF membrane (Millipore, Billerica, USA) in NuPAGE transfer buffer (Invitrogen, Paisley, UK). Membranes were blocked and incubated with antibodies in 5\% dried milk in TBS-T (Tris-buffered saline$0.1 \%$ Tween 20) and were washed in TBS-T. Primary antibodies: rabbit monoclonal anti-phosphoThr37/46 4E-BP1, 1:500, and rabbit polyclonal anti-4E-BP1, 1:500 (\#2855 and \#9452, Cell Signalling Technology, Beverly, USA); mouse monoclonal anti-eIF4E, 1:500 (sc9976, Santa Cruz, USA). We have previously validated specificities of these antibodies, including the phospho-specificity of the anti-phospho clone [31], although we cannot exclude that the anti-phospho-4EBP1 may cross-react with phospho-4E-BP2 or 3. Secondary antibodies: anti-mouse/rabbit HRP conjugates, 1:1000 (DAKO, Glostrup, Denmark). Proteins were detected using Supersignal West Femto (Thermo Fisher, Waltham, USA) and Chemidoc XRS (BioRad, Hercules, USA), and analysed using ImageJ $1.42 \mathrm{q}$ (NIH, http://rsb.info.nih.gov/ij).

\section{Translational efficiency assay}

We have previously reported the protocol in considerable detail $[32,33]$. In brief, relative GFP protein and mRNA levels were used to calculate amounts of GFP protein produced per unit mRNA. RNA was purified using RNeasy (Qiagen, Crawley, UK) and contaminating DNA was removed with TURBO DNase I (Applied Biosystems, Warrington, UK). cDNA was synthesized from oligo-dT primed RNA using Superscript II (Invitrogen, Paisley, UK). Real-time PCR reactions were performed in triplicate using SYBR Green PCR Master Mix on an ABI7900HT machine (Applied Biosystems, Warrington, 
UK). GFP mRNA levels were normalized to those of the reference gene RPLP0 [34] and relative expression calculated using the $\Delta \Delta \mathrm{Ct}$ method [35]. For analysis of GFP protein expression, cells were suspended in media containing $1 \%$ serum and fluorescence quantified (mean fluorescent intensity of $10^{4}$ events after exclusion of debris/dead cells on forward activated light scatter/side scatter) at $525 \mathrm{~nm}$ using an LSRII machine (BD Biosciences, Oxford, UK).

\section{Ethical issues, patient material, immunohistochemistry}

Ethical permissions were obtained from Northern and Yorkshire MREC (4/MRE03/89) and Leeds East REC (05/Q1206/136). Postmenopausal female patients with operable early breast cancer (T1-3, N0-1, M0) proceeding to primary surgery were recruited, written informed consent was taken, and patients were treated as previously described in detail [36]. In brief, core biopsies were taken at time of presentation with a palpable breast lump and were processed for diagnostic assessments. Patients were given 11-14 days of everolimus $5 \mathrm{mg}$ once daily immediately before tumour resection. Excision specimens were processed by the pathology laboratory for diagnostic tests. Clinical/pathological details of patients are listed elsewhere [36]. Matched biopsy and excisional tumour blocks from 22 patients were used. Immunohistochemistry was performed for Ki67 (mouse monoclonal clone MIB-1; Dako, Glostrup, Denmark), 4E-BP2 (rabbit polyclonal \#2845, Cell Signalling Technology, Beverly, USA), eIF4E, 4E-BP1 and phosphoThr37/46 4E-BP1 (as for Westerns) exactly as described and validated previously $[31,36]$ on single sections from each case for each antigen. Ki67 was quantified using a previously validated protocol for scoring percentages of stained cells as proportions of total cancer cells [37], and these data have been published [36]. Other markers were scored by two independent individuals (VJC, CS) taking into account average intensity and percentage of positively stained tumour cells. Intensity scores ( 0 no staining, 1 weak, 2 moderate and 3 strong) were added to percentages scores $(1<5 \%, 26$ $25 \%, 326-75 \%$ and $4>75 \%$ ) giving totals of 0 or $2-7$. Consensus scores were determined for sections with different initial scores; scoring was overseen by a consultant breast histopathologist (AMH).

\section{Statistics}

Analyses were performed using Student's T Test, Spearman's rho correlation, or linear regression in Excel v12 (Microsoft, Redmond, USA), SPSS v15 (SPSS, Chicago, USA) and MATLAB (MathWorks, Natick, USA). Tests were two sided; $\mathrm{p}<0.05$ was considered to indicate significance.

\section{Results}

Cell lines show a range of sensitivities to rapamycin

Rapamycin and its derivatives induce a broad range of responses when used as cancer therapeutics with growth of some cancers reduced while others are resistant. We were interested to examine this variation, therefore we treated a panel of cell lines with rapamycin and determined drug sensitivities. The panel was representative of the three most common cancers in the UK: colorectal (SW480 and Caco2, moderately-differentiated and heterogeneous colon cancer lines respectively); lung (U2020, small cell, and H1299 and A549, non small cell cancer lines); and breast (MCF7 and MDA-MB-231, luminal and basal breast cancer lines respectively). In addition, two immortal breast epithelial lines of noncancer origin (HB2 and MCF10A) were examined in order to allow study of potential differential sensitivity between cancer and non-cancer cells. We treated cells with doses of rapamycin and determined proliferation/ survival relative to control treated cells using MTT assays after $48 \mathrm{~h}$ (Figure 1A). Sensitivities to the highest dose are shown in Figure 1B. As expected a range of sensitivities were seen, with a three fold difference between the most sensitive (MCF7) and most resistant (MDA-MB-231). Cells of non-cancer origin (HB2 and MCF10A) were found to have sensitivities between these extremes.

\section{The phosphorylation state of 4E-BP1 does not predict rapamycin sensitivity}

Next, we aimed to identify molecular markers that correlated with these sensitivities, therefore that might represent predictive biomarkers for mTOR inhibitors. Potential biomarkers have previously been proposed; of particular interest was the phosphorylation status of $4 \mathrm{E}$ BP1 [24] since the 4E-BP1/eIF4E axis has been shown to be critical for mTOR-mediated transformation [38]. 4E-BP1 is directly phosphorylated by mTORC1 [12], potentially leading to increased eIF4E activity and enhanced translation of cancer-related transcripts [15-17]. Thus, levels of phosphorylated 4E-BP1 may reflect contributions of mTORC1-signalling to cancerassociated translational deregulation, and consequently the sensitivity of such deregulated cells to mTOR inhibition. We performed Western blot analysis of levels of mTORC1-dependent 4E-BP1 phosphorylation (Thr37/ Thr46) in the same cell lines as before (Figure 2A). At least three different phosphorylated 4E-BP1 (phospho$4 \mathrm{E}-\mathrm{BP} 1$ ) species were seen, representing various combinations of the seven potential phosphorylation events [39-41]. We found no correlation between phospho-4EBP1 and rapamycin sensitivity (compare Figures 2A and $1 \mathrm{~B})$. However, levels of phospho-4E-BP1 reflect not 


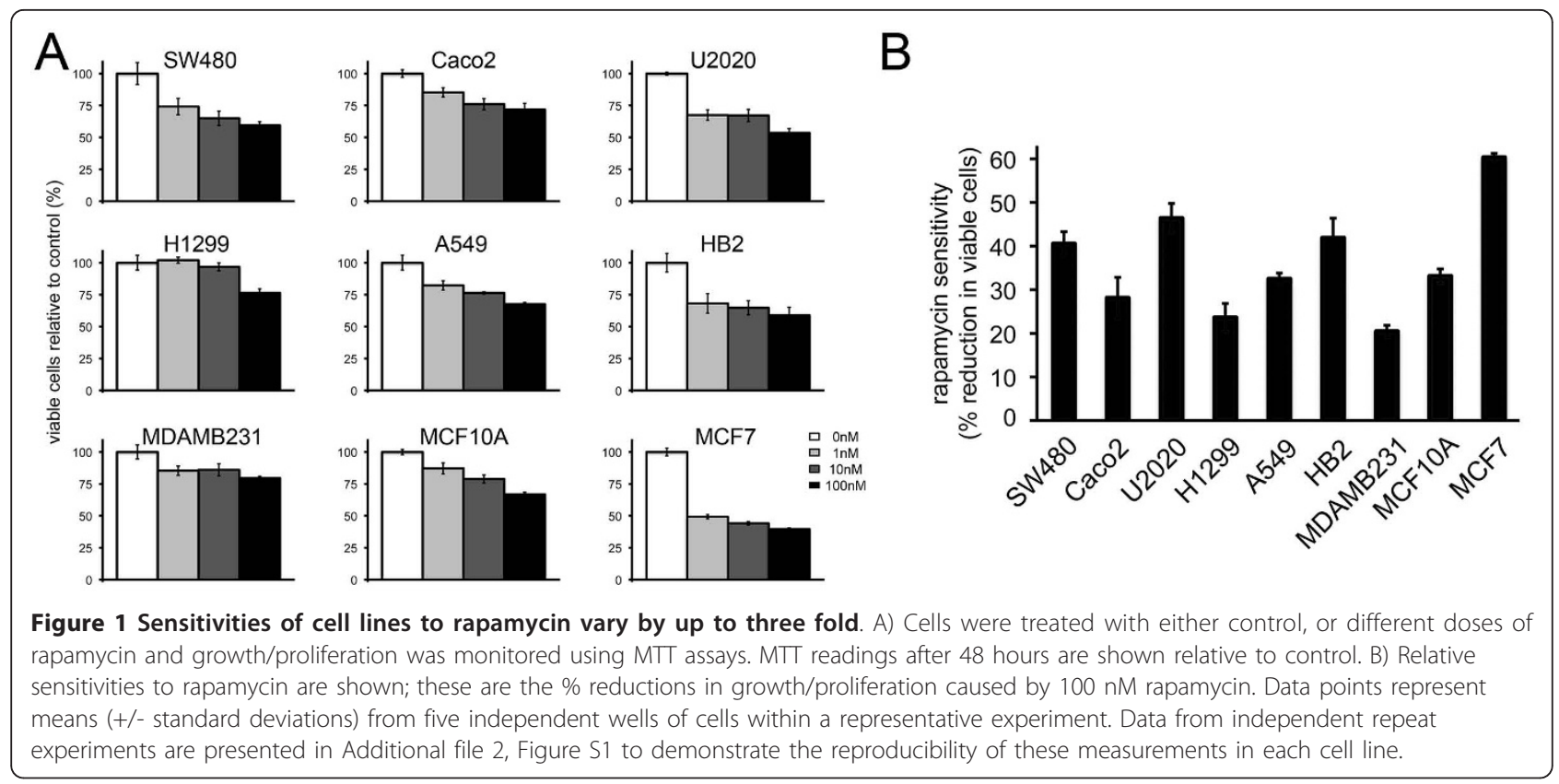

only mTORC1 activity but also levels of overall 4E-BP1, therefore we also analysed total 4E-BP1 expression (Figure $2 \mathrm{~B}$ ) and determined the ratios of phospho- to total $4 \mathrm{E}-\mathrm{BP} 1$ (Figure 2C) as a measure of mTORC1's influence on 4E-BP1 function, as previously reported [25]. We found no correlation between this measure and rapamycin sensitivity. Finally, 4E-BP1's influence on cellular behaviour is determined by the amount of eIF4E remaining unbound by $4 \mathrm{E}-\mathrm{BP} 1$, consequently, variation

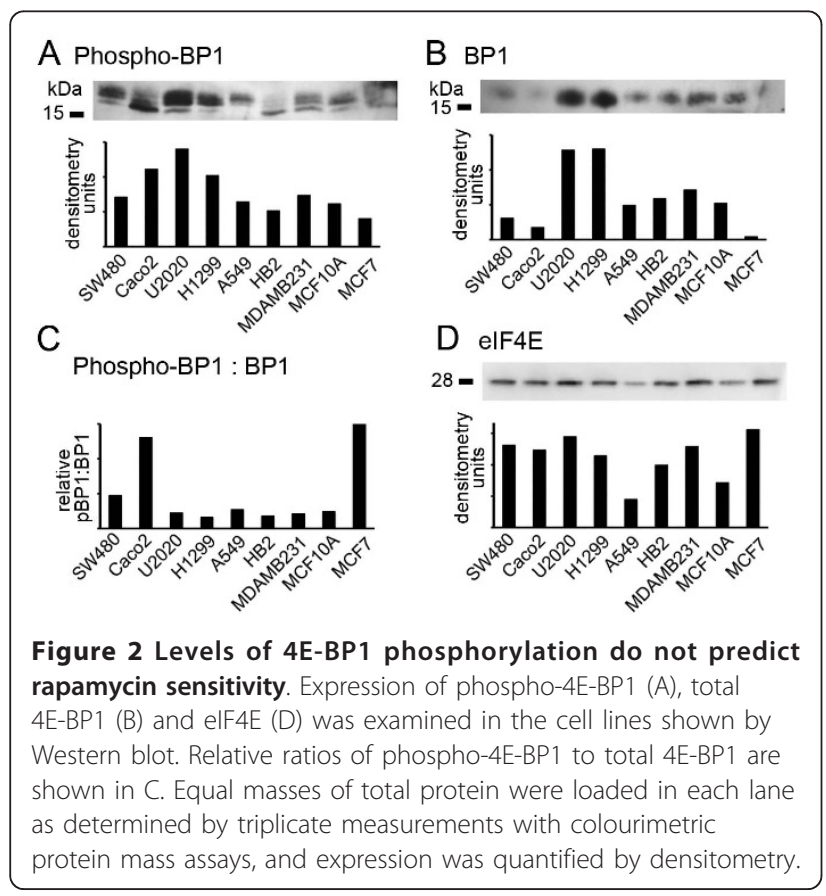

in eIF4E expression would also have a critical role. We analysed eIF4E expression and found greater than three fold variation in eIF4E expression (Figure 2D). We concluded that levels of phospho-4E-BP1 do not correlate with functional influences of mTORC1 on capdependent translation, partly as substantial variations in total 4E-BP1 and eIF4E expression mask this direct relationship. A measure of eIF4E activity would, however, take into account the variation in expression of all these components and might provide a better predictive marker.

\section{Assessment of translational efficiencies defined by a structured 5'UTR; estimation of elF4E activity}

Our hypothesis was that assessment of the activity of a key mTORC1 regulated pathway gives direct insights into the contribution of deregulated mTORC1 to cellular behaviour and therefore, potentially, into likely sensitivity to mTOR-inhibitors. A critical effect of upregulated mTORC1 is to up-regulate eIF4E activity thereby enhancing translational efficiencies of transcripts with structured 5'UTRs [15]; therefore, we designed an assay to measure these translational efficiencies. We have previously shown that a 5 'UTR from human axin2 transcripts contains a sixty nucleotide sequence that is predicted to form a stable stem-loop structure [29]. This sequences meets the criteria associated with UTRs that determine differential translational efficiencies in response to changes in eIF4E activity [16], while lacking other translation regulatory motifs (e.g. upstream AUG codons or binding sites for trans-acting proteins). In addition, we previously demonstrated that this sequence 
determined cell type specific translational efficiencies [29]. We now wished to examine whether the translational efficiency defined by this sequence would respond to changes in eIF4E activity, and could therefore be representative of mTORC1's influence on cap-dependent translation of structured transcripts. The sequence was cloned upstream of the GFP reading frame in an expression vector. MCF7 cells were transiently transfected with an equal copy number of vectors to allow expression of GFP mRNAs with either a control non-regulatory 5'UTR or this sequence as a 5'UTR, along with either empty expression plasmids or plasmids allowing eIF4E over-expression. GFP protein expression was measured by flow-cytometry and GFP mRNA expression was measured by qPCR allowing determination of relative translational efficiencies for each GFP message as previously described [32,33] (Figure 3A). Western blot analyses were used to confirm expression of exogenous eIF4E and GFP (Figure 3B). The translational efficiency of the control reporter was not significantly altered by eIF4E over-expression (compare lanes 1 and 2), demonstrating that eIF4E over-expression did not cause a general enhancement of translation. As previously reported [29], the structured 5'UTR conferred repression of translation (compare lanes 1 and 3; p = 0.002). Critically, this repression was overcome by exogenous eIF4E (compare lanes 3 and $4 ; \mathrm{p}=0.002$ ), resulting in translation with the same efficiency as messages lacking inhibitory 5'UTRs. We concluded that this reporter did indeed respond to changes in eIF4E activity and thus can be used to provide an estimate of eIF4E-dependent translation from structured 5'UTRs.

Estimates of elF4E activity predict rapamycin sensitivity in tissue culture cells

Relative translational efficiencies specified by this eIF4Eresponsive 5'UTR were determined in the panel of cell lines. Cells were transiently transfected with vectors to allow expression of GFP mRNAs with control or the structured 5'UTR as before, and translational efficiencies were determined (Figure 4A). A range of translational efficiencies was seen, with A549 cells determining the lowest, and HB2 cells the highest (Figure 4B). Surprisingly, the two cells lines of non-cancer origin (HB2 and MCF10A) were found to determine relatively efficient translation from the reporter 5'UTR. Importantly, translational efficiencies specified by the eIF4E-responsive 5'UTR correlated with rapamycin sensitivity (Figure 5). Initially, we analysed this relationship using Spearman's rank correlation coefficient; we found a strong and significant positive association $(r=0.72 ; \mathrm{p}=0.037)$.

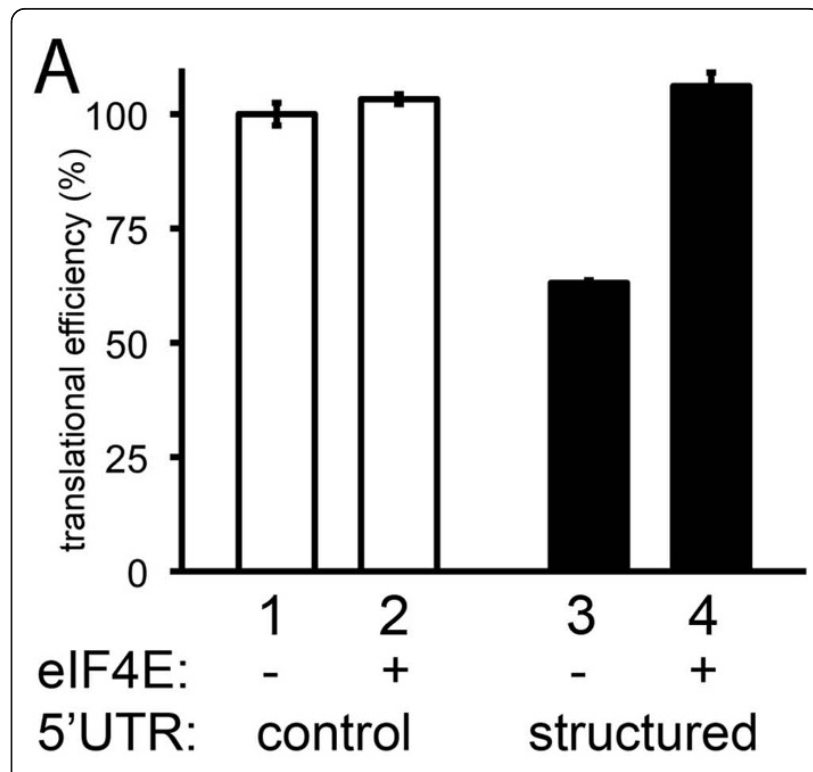

$B$
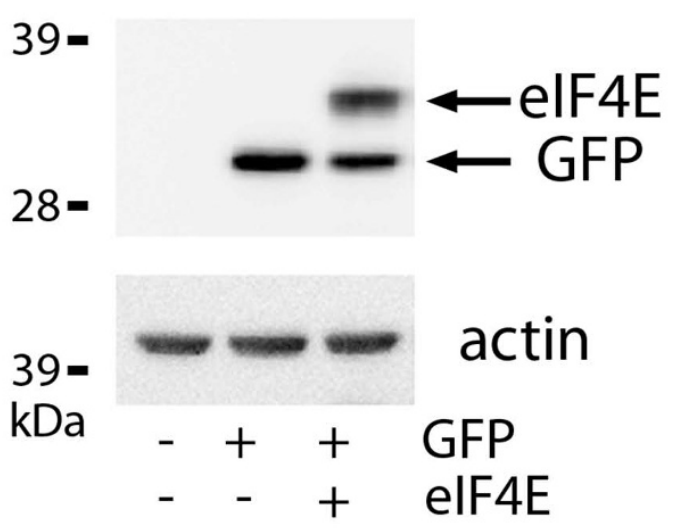

Figure 3 Translational efficiency specified by a structured $5^{\prime}$ UTR reporter responds to changes in elF4E activity. A) Reporters were constructed to express mRNAs containing the GFP reading frame preceded by a control 5'UTR lacking regulatory motifs (control) or a sequence predicted to form a stable stem-loop structure (structured). MCF7 cells were transiently transfected with equal copy numbers of either control or structured reporters along with either empty expression vector (-) or vector to allow overexpression of elF4E (+). GFP protein and mRNA were quantified by flow-cytometry and real-time PCR respectively. Translational efficiency (protein synthesised per unit mRNA) is presented relative to the control. Data points represent means (+/-standard deviations) of values from three separate wells of cells within a representative experiment. B) Expression of exogenous proteins was confirmed within cells transfected with control GFP reporter and vector to allow over-expression of elF4E as shown by Western blot analysis for the HA-epitope tag; exogenous GFP and elF4E both include this epitope. Note that the small reduction in GFP protein associated with elF4E-transfection does not result from a change in translational efficiency (see Panel A). 

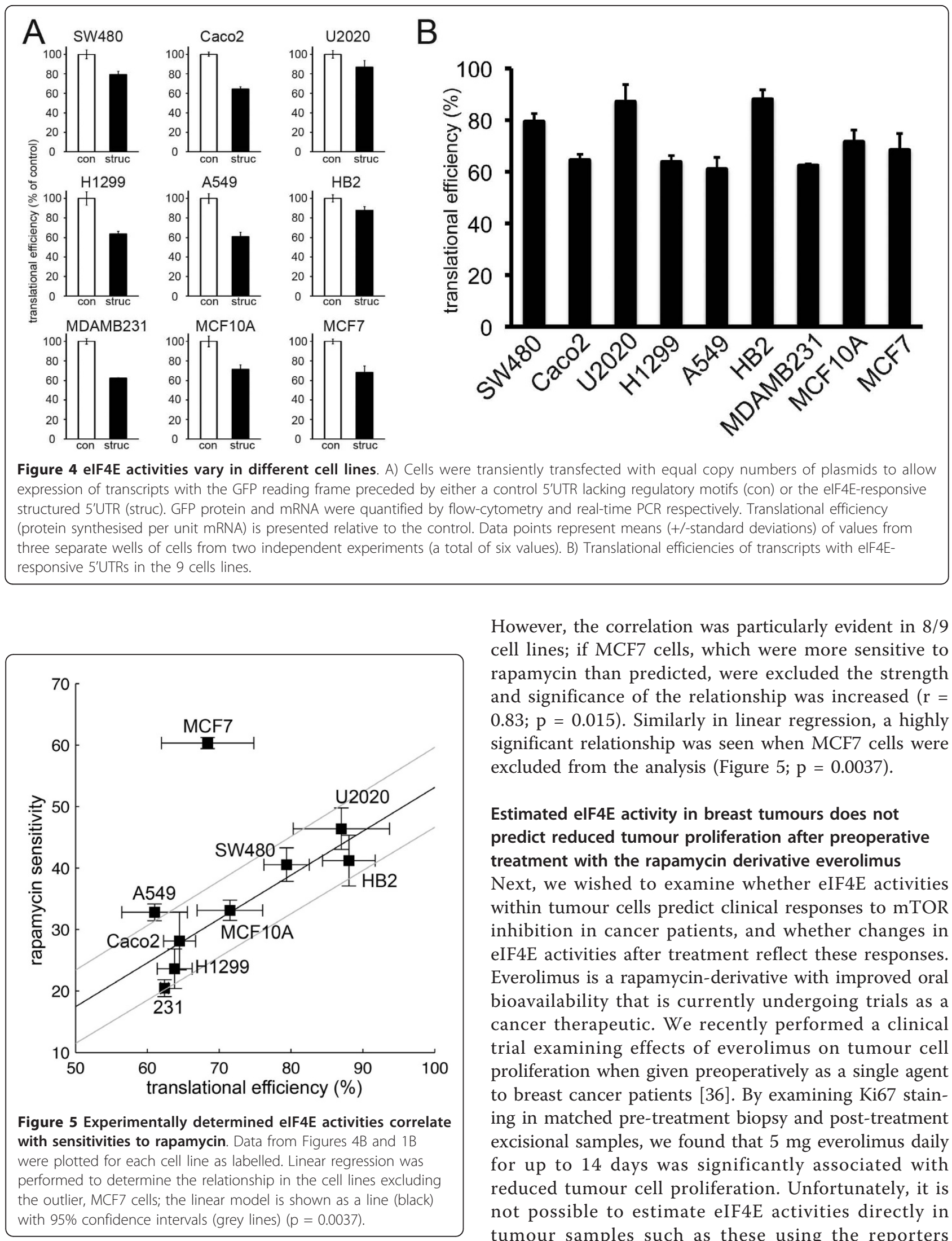

However, the correlation was particularly evident in 8/9 cell lines; if MCF7 cells, which were more sensitive to rapamycin than predicted, were excluded the strength and significance of the relationship was increased $(\mathrm{r}=$ $0.83 ; \mathrm{p}=0.015)$. Similarly in linear regression, a highly significant relationship was seen when MCF7 cells were excluded from the analysis (Figure $5 ; \mathrm{p}=0.0037$ ).

Estimated elF4E activity in breast tumours does not predict reduced tumour proliferation after preoperative treatment with the rapamycin derivative everolimus Next, we wished to examine whether eIF4E activities within tumour cells predict clinical responses to mTOR inhibition in cancer patients, and whether changes in eIF4E activities after treatment reflect these responses. Everolimus is a rapamycin-derivative with improved oral bioavailability that is currently undergoing trials as a cancer therapeutic. We recently performed a clinical trial examining effects of everolimus on tumour cell proliferation when given preoperatively as a single agent to breast cancer patients [36]. By examining Ki67 staining in matched pre-treatment biopsy and post-treatment excisional samples, we found that $5 \mathrm{mg}$ everolimus daily for up to 14 days was significantly associated with reduced tumour cell proliferation. Unfortunately, it is not possible to estimate eIF4E activities directly in tumour samples such as these using the reporters 
described above. As an alternative we estimated eIF4E activity from expression and phosphorylation states of multiple regulators of the translational initiation pathway (Figure 6; Table 1). We have previously described the development and prognostic value of this estimate in breast tumours [31]. Expression levels of eIF4E, 4EBP1, 4E-BP2 and Thr37/Thr46 phosphorylated 4E-BP1 (phospho-4E-BP1) within tumour cells were determined semi-quantitatively in matched pre- and post-treatment tumour samples from 22 patients using immunohistochemistry. Activity of eIF4E was estimated by combining these scores to reflect the inhibitory influence of 4E-BPs on eIF4E, and the activating influence of 4E-BP1 phosphorylation on eIF4E, using a formula previously derived from regression modelling of individual contributions of each component to prognosis in a large cohort of breast cancer patients: X-B1/4+PB1/2-B2/4, where X, B1, PB1 and $\mathrm{B} 2$ represent scores for eIF4E, 4E-BP1 phospho-4EBP1 and 4E-BP2 respectively [31]. We previously showed that this measure gave improved insights into the prognostic influence of eIF4E by reflecting eIF4E activity more accurately than examining eIF4E expression levels alone.

17/22 tumours showed reduced Ki67 scores after treatment (mean reduction 48\%) indicating apparent

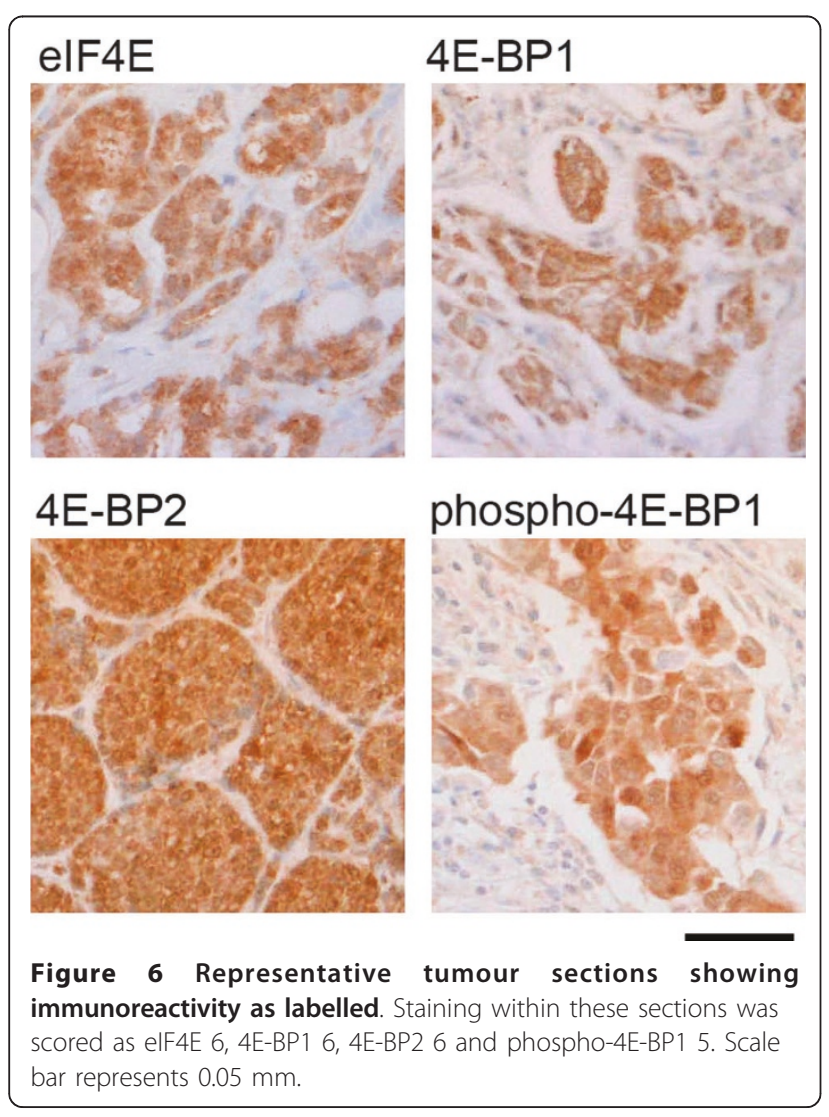

responses to everolimus (Table 1). Disappointingly, estimates of pre-treatment eIF4E activity did not predict the occurrence or extent of these responses. Similarly, pre-treatment phospho-4E-BP1 levels had no predictive value. Estimated eIF4E activity was, however, reduced in post-treatment samples (mean change in score -1.7; range -8 to $+1.25 ; \mathrm{p}<0.001$ ) but surprisingly this was not attributable to reduced phospho-4E-BP1. Phospho4E-BP1 expression was reduced after treatment (mean -2.3 ; range -6 to $+1 ; \mathrm{p}<0.001$ ), suggesting a reduction in mTOR-dependent phosphorylation of $4 \mathrm{E}-\mathrm{BP} 1$, but this reduction in phospho-4E-BP1 was not significantly correlated with the reduction in estimated eIF4E activity, and changes in levels of the other components had strong influences on estimated eIF4E activity. For example, 4E-BP1 expression changed considerably (mean -0.3 ; range -5 to $+5 ; \mathrm{p}=0.01$ ), meaning that 8 individual decreases in phospho-4E-BP1 could be explained at least partially by reductions in total $4 \mathrm{E}-\mathrm{BP} 1$, as opposed to reduced phosphorylation. This explanation is supported by observations that the phospho-4E-BP1 species examined here (Thr37/46) can be relatively resistant to mTOR inhibition [39]. 4E-BP2 expression also frequently changed (mean +2.2 ; range -2 to +7 ; $<<0.001$ ), while some individuals showed dramatic changes in expression of eIF4E (mean - 0.1 ; range -6 to +4 ). Interestingly changes in eIF4E and 4E-BP1 were positively associated $(\mathrm{r}=0.60, \mathrm{p}=0.003)$, often resulting in relatively small changes in estimated eIF4E activity, despite substantial fluctuations in expression of the individual proteins. Critically, neither reduced estimated eIF4E activity or reduced phospho-4E-BP1 correlated with reduced $\mathrm{Ki} 67$.

\section{High estimated elF4E activities in breast tumours are associated with everolimus-induced changes in elF4E regulation}

A striking observation was that post-treatment levels of eIF4E and the 4E-BPs frequently varied considerably from pre-treatment levels. Our hypothesis was that these changes represent development of acquired resistance to inhibition of eIF4E activity, by induction of changes in eIF4E regulation within tumour cells or by clonal selection of cells with different eIF4E-related expression profiles. Importantly, this proposed acquired resistance is not necessarily reflected in higher proliferation rates. Using this hypothesis, one would predict that tumours with high pre-treatment estimated eIF4E activities would be most subject to drug-induced expression changes or to clonal selection pressures, and would show the greatest adaptive changes in eIF4E regulation. In fact, high estimated eIF4E activity pre-treatment did positively correlate with the combined magnitude of changes in expression 
Table 1 Expression scores for elF4E (4E), 4E-BP1 (BP1), 4E-BP2 (BP2), phospho-4E-BP1 (pBP1) and Ki67, and estimates of eIF4E activity in matched pre- and post-treatment samples from patients treated with 11-14 days of everolimus

\begin{tabular}{|c|c|c|c|c|c|c|c|c|c|c|c|c|}
\hline \multirow[b]{2}{*}{ Patient } & \multicolumn{6}{|c|}{ pre-treatment } & \multicolumn{6}{|c|}{ post-treatment } \\
\hline & $4 \mathrm{E}$ & BP1 & BP2 & pBP1 & activity & Ki67 & $4 \mathrm{E}$ & BP1 & BP2 & pBP1 & activity & Ki67 \\
\hline 1 & 6 & 5 & 5 & 6 & 6.5 & 15.5 & 0 & 0 & 6 & 0 & -1.5 & 23.1 \\
\hline 2 & 3 & 5 & 5 & 5 & 3 & 6.0 & 0 & 3 & 5 & 0 & -2 & 0.9 \\
\hline 3 & 3 & 3 & 5 & 4 & 3 & 7.6 & 5 & 4 & 5 & 3 & 4.25 & 2.7 \\
\hline 4 & 5 & 6 & 6 & 6 & 5 & 22.1 & 6 & 7 & 7 & 6 & 5.5 & 16.7 \\
\hline 5 & 4 & 6 & 4 & 6 & 4.5 & 44.6 & 4 & 4 & 4 & 3 & 3.5 & 29.7 \\
\hline 6 & 4 & 0 & 6 & 6 & 5.5 & 34.2 & 5 & 5 & 5 & 4 & 4.5 & 23.0 \\
\hline 7 & 3 & 6 & 5 & 5 & 1.75 & 4.2 & 4 & 4 & 4 & 0 & 2 & 2.7 \\
\hline 8 & 4 & 3 & 4 & 2 & 3.25 & 1.6 & 3 & 0 & 4 & 3 & 3.5 & 4.4 \\
\hline 9 & 5 & 7 & 7 & 6 & 4.5 & 15.3 & 5 & 6 & 7 & 5 & 4.25 & 6.8 \\
\hline 10 & 5 & 7 & 6 & 5 & 4.25 & 22.6 & 5 & 3 & 6 & 2 & 3.75 & 6.5 \\
\hline 11 & 5 & 0 & 6 & 2 & 4.5 & 18.4 & 5 & 0 & 4 & 0 & 4 & 7.4 \\
\hline 12 & 5 & 3 & 6 & 6 & 5.75 & 3.6 & 6 & 6 & 7 & 3 & 4.25 & 47.2 \\
\hline 13 & 4 & 4 & 0 & 5 & 5.5 & 10.1 & 6 & 5 & 5 & 2 & 4.5 & 3.6 \\
\hline 14 & 6 & 4 & 0 & 6 & 8 & 17.4 & 4 & 4 & 6 & 2 & 2.5 & 10.1 \\
\hline 15 & 5 & 0 & 0 & 6 & 8 & 10.2 & 4 & 0 & 7 & 5 & 4.75 & 17.8 \\
\hline 16 & 5 & 4 & 0 & 4 & 6 & 10.6 & 6 & 4 & 6 & 2 & 4.5 & 13.0 \\
\hline 17 & 4 & 4 & 2 & 6 & 5.5 & 16.2 & 6 & 3 & 5 & 4 & 6 & 8.4 \\
\hline 18 & 0 & 0 & 0 & 4 & 2 & 6.2 & 4 & 2 & 7 & 0 & 1.75 & 5.2 \\
\hline 19 & 4 & 4 & 0 & 5 & 5.5 & 18.2 & 7 & 7 & 5 & 3 & 5.5 & 9.0 \\
\hline 20 & 6 & 4 & 0 & 7 & 8.5 & 18.0 & 5 & 6 & 7 & 5 & 4.25 & 3.8 \\
\hline 21 & 5 & 4 & 6 & 6 & 5.5 & 20.0 & 0 & 0 & 5 & 5 & 1.25 & 13.4 \\
\hline 22 & 5 & 4 & 2 & 4 & 5.5 & 6.8 & 4 & 4 & 7 & 3 & 2.75 & 5.3 \\
\hline
\end{tabular}

of the four markers $(\mathrm{r}=0.46, \mathrm{p}=0.03)$ and, in particular, with increases in 4E-BP2 expression $(r=0.55$, $\mathrm{p}<0.01)$. We concluded that high estimated eIF4E activity may, in fact, predict tumour response to everolimus; however this response is not the expected reduction in proliferation, but is development of changes in eIF4E regulation, presumably to promote everolimus resistance.

\section{Discussion}

The mTOR pathway, which promotes cell proliferation, presents an attractive target for cancer therapy since it is deregulated in a wide range of cancer types and a large proportion of cases of each type [2]. However, resistance of some cancers to $\mathrm{mTOR}$-directed therapeutics has limited the success of mTOR inhibitors. We have examined this variation in response, initially, in cell lines. As expected, and in accordance with other published work [42-44], we found a range of sensitivities to rapamycin (Figure 1). Surprisingly, we found that cancer cell lines were not more sensitive than cells of noncancer origin, despite the well established preferential sensitivity of cancer cells over non-cancer cells in animal models and, to an extent, in humans [3-5]. This observation most likely demonstrates that up-regulation of
mTORC1, and consequently sensitivity to its inhibition, is actually associated with growth as opposed to malignancy, and therefore that highly-proliferative, immortal, non-cancer cell lines are un-representative of 'normal', relatively slowly growing, epithelial cells with respect to mTORC1 signalling. The efficacy of rapamycin as an immunosuppressant drug [1] and the side-effects seen in cancer therapies $[21,22]$ support the view that proliferating cells are targeted.

Identification of predictive biomarkers for mTORtargeted therapies such as rapamycin or everolimus has become a research focus [2]. Levels of phosphorylated mTOR, S6K1 or 4E-BP1 have been seen as logical markers as these phosphorylation events induce mTOR activity or are directly catalysed by mTORC1, and therefore levels may reflect the extent of mTORC1 deregulation. However, in principle, it is obvious that levels of these species may not correlate directly with their influences on down-stream signalling and consequent changes in cellular behaviour, since these influences would also be defined by expression/activity of the other regulatory molecules of the pathways. Despite this, some predictive value has been demonstrated for each marker $[23,24,27]$. We found levels of phosphorylated 4E-BP1, and the proportions of phospho-4E-BP1 within the total 
pool of 4E-BP1 to be unrelated to rapamycin sensitivity in tissue culture, in accordance with previous work in a cell line panel also containing MCF7 and MDA-MB-231 cells [42]. Moreover, we showed that this, in fact, was the expected result in the context of variation in 4E-BP1 and eIF4E expression (Figure 2). As an alternative predictive marker, we developed assays to estimate one of the key functional end-points of mTORC1 signalling, eIF4E activity. We found this estimate to be significantly associated with rapamycin sensitivity in cell culture. It was notable, however, that estimated eIF4E activity was the most significant predictor of rapamycin sensitivity for 8 of the cell lines (Figure 5), while MCF7 cells were twice as sensitive as predicted by this relationship. MCF7 cells over-express S6K1, on account of amplification of its gene [45]; one explanation for enhanced sensitivity in MCF7 cells may be that with constitutively high S6K1 activity, the cells are dependent upon mTOR-induced S6K functions such as more general translational effects [46]. In support of this, S6K1 overexpression has previously been associated with increased rapamycin sensitivity [42].

Importantly, we also examined whether estimates of pre-treatment eIF4E activity in clinical breast tumours predicted response to the mTOR inhibitor everolimus. Disappointingly and in contrast to our in vitro work, we found estimated eIF4E activity did not predict response to mTOR inhibition as assessed by change in tumour cell proliferation. However, we did find that pretreatment eIF4E activity in tumours was significantly associated with substantial changes in the expression of eIF4E and its regulators post-treatment. We interpret this to suggest that cancers with high eIF4E activity may indeed have been sensitive to everolimus, as suggested by our in vitro data, but that the cells remaining after two weeks of drug treatment reflect selection to acquire drug resistance by changing the pathways regulating eIF4E function. Data show that this proposed resistance is not necessarily associated with lower estimated eIF4E activity or higher proliferative rates. This hypothesis highlights a difference between short-term (two day) sensitivity assays in vitro and longer term (two week) drug treatments in patients; in the latter case it is inevitably more difficult to assess the early response of tumour cells to treatment and there is considerable scope for acquired changes to take place. Finally, it is interesting to note that our data do not support the use of phospho-4E-BP1 as either a predictive or pharmacodynamic marker for mTOR inhibitors as some have attempted $[2,21]$ since it is clear that changes in phospho-4E-BP1 relate not only to inhibition of 4E-BP1 phosphorylation, but also to dramatic changes in overall 4E-BP1 expression.

\section{Additional material}

Additional file 1: Table S1. Cell culture and transfection conditions.

Additional file 2: Figure S1. Sensitivities of cell lines to rapamycin are reproducible. Relative sensitivities to $100 \mathrm{nM}$ rapamycin are shown; these are the $\%$ reductions in growth/proliferation caused by the drug as compared to control treated cells. Data from Figure 1B are reproduced (filled bars) alongside independent repeat analyses (open bars). Data points represent means (+/- standard deviations) from five independent wells of cells.

\section{Acknowledgements}

We thank the United States Department of Defence, Yorkshire Cancer Research and Novartis Pharma for funding.

\section{Author details}

${ }^{1}$ Leeds Institute of Molecular Medicine, St. James's University Hospital, Leeds University, Leeds, LS9 7TF, UK. Department of Histopathology, St. James's University Hospital, Leeds, LS9 7TF, UK. ${ }^{3}$ University of Edinburgh Cancer Research Centre, Institute of Genetics \& Molecular Medicine, Edinburgh, EH4 2XR, UK. ${ }^{4}$ Edinburgh Breakthrough Research Unit, Institute of Genetics \& Molecular Medicine, University of Edinburgh, Edinburgh, EH4 2XR, UK. ${ }^{5}$ Department of Applied Mathematics and Theoretical Physics, Cambridge University, Cambridge, CB2 OWA, UK.

\section{Authors' contributions}

SS, VJC, LJC and NI performed the in vitro experiments. BM and JNM performed statistical analyses. CABS, VJC and AMH performed semiquantitative scoring of tissue sections. VSS, EJM, JMSB, JMD established the everolimus clinical trial, and obtained/analysed clinical samples. TAH devised and directed the study. All authors contributed to writing the manuscript and have approved the final version.

\section{Competing interests}

JMD possesses an unrestricted educational grant and honoraria for seminar presentations from Novartis Pharma. The other authors declare that they have no conflicts of interest.

Received: 15 November 2010 Accepted: 14 February 2011 Published: 14 February 2011

\section{References}

1. Mulay AV, Cockfield S, Stryker R, Fergusson D, Knoll GA: Conversion from calcineurin inhibitors to sirolimus for chronic renal allograft dysfunction: a systematic review of the evidence. Transplantation 2006, 82:1153-1162.

2. Meric-Bernstam F, Gonzalez-Angulo AM: Targeting the mTOR signaling network for cancer therapy. J Clin Oncol 2009, 27:2278-2287.

3. Hudes G, Carducci M, Tomczak P, Dutcher J, Figlin R, Kapoor A, Staroslawska E, Sosman J, McDermott D, Bodrogi I, et al: Temsirolimus, interferon alfa, or both for advanced renal-cell carcinoma. $N$ Engl J Med 2007, 356:2271-2281.

4. Motzer RJ, Escudier B, Oudard S, Hutson TE, Porta C, Bracarda S, Grunwald V, Thompson JA, Figlin RA, Hollaender N, et al: Efficacy of everolimus in advanced renal cell carcinoma: a double-blind, randomised, placebo-controlled phase III trial. Lancet 2008, 372:449-456.

5. Stallone G, Schena A, Infante B, Di Paolo S, Loverre A, Maggio G, Ranieri E, Gesualdo L, Schena FP, Grandaliano G: Sirolimus for Kaposi's sarcoma in renal-transplant recipients. N Engl J Med 2005, 352:1317-1323.

6. Oshiro N, Yoshino K, Hidayat S, Tokunaga C, Hara K, Eguchi S, Avruch J, Yonezawa K: Dissociation of raptor from mTOR is a mechanism of rapamycin-induced inhibition of mTOR function. Genes Cells 2004, 9:359-366.

7. Chen J, Zheng XF, Brown EJ, Schreiber SL: Identification of an 11-kDa FKBP12-rapamycin-binding domain within the 289-kDa FKBP12rapamycin-associated protein and characterization of a critical serine residue. Proc Natl Acad Sci USA 1995, 92:4947-4951. 
8. Shor B, Zhang WG, Toral-Barza L, Lucas J, Abraham RT, Gibbons JJ, Yu K: A new pharmacologic action of $\mathrm{CCl}-779$ involves FKBP12-independent inhibition of mTOR kinase activity and profound repression of global protein synthesis. Cancer Res 2008, 68:2934-2943.

9. Dowling RJ, Topisirovic I, Fonseca BD, Sonenberg N: Dissecting the role of mTOR: Lessons from mTOR inhibitors. Biochim Biophys Acta 2010, 1804:433-439.

10. Shaw RJ, Bardeesy N, Manning BD, Lopez L, Kosmatka M, DePinho RA, Cantley LC: The LKB1 tumor suppressor negatively regulates mTOR signaling. Cancer Cell 2004, 6:91-99.

11. Feng $Z$, Zhang $H$, Levine $A J$, Jin S: The coordinate regulation of the $\mathrm{p} 53$ and mTOR pathways in cells. Proc Natl Acad Sci USA 2005, 102:8204-8209.

12. Hara K, Yonezawa K, Kozlowski MT, Sugimoto T, Andrabi K, Weng QP, Kasuga M, Nishimoto I, Avruch J: Regulation of elF-4E BP1 phosphorylation by mTOR. J Biol Chem 1997, 272:26457-26463.

13. von Manteuffel SR, Dennis PB, Pullen N, Gingras AC, Sonenberg N, Thomas G: The insulin-induced signalling pathway leading to $S 6$ and initiation factor $4 \mathrm{E}$ binding protein 1 phosphorylation bifurcates at a rapamycin-sensitive point immediately upstream of p70s6k. Mol Cell Biol 1997, 17:5426-5436.

14. Beretta L, Gingras AC, Svitkin $Y$, Hall MN, Sonenberg N: Rapamycin blocks the phosphorylation of $4 \mathrm{E}-\mathrm{BP} 1$ and inhibits cap-dependent initiation of translation. E M B O J 1996, 15:658-664.

15. Koromilas AE, Lazaris-Karatzas A, Sonenberg N: mRNAs containing extensive secondary structure in their $5^{\prime}$ non-coding region translate efficiently in cells overexpressing initiation factor elF-4E. E M B O J 1992, 11:4153-4158.

16. Gray NK, Hentze MW: Regulation of protein synthesis by mRNA structure. Mol Biol Rep 1994, 19:195-200.

17. Kozak M: An analysis of vertebrate mRNA sequences: intimations of translational control. J Cell Biol 1991, 115:887-903.

18. Culjkovic B, Topisirovic I, Borden KL: Controlling gene expression through RNA regulons: the role of the eukaryotic translation initiation factor elF4E. Cell Cycle 2007, 6:65-69.

19. Culjkovic B, Topisirovic I, Skrabanek L, Ruiz-Gutierrez M, Borden KL: elF4E promotes nuclear export of cyclin D1 mRNAs via an element in the 3'UTR. J Cell Biol 2005, 169:245-256.

20. Raught B, Peiretti F, Gingras AC, Livingstone M, Shahbazian D, Mayeur GL, Polakiewicz RD, Sonenberg N, Hershey JW: Phosphorylation of eucaryotic translation initiation factor $4 \mathrm{~B}$ Ser422 is modulated by S6 kinases. E M B O J 2004, 23:1761-1769.

21. Tabernero J, Rojo F, Calvo E, Burris H, Judson I, Hazell K, Martinelli E, Ramon y Cajal S, Jones S, Vidal L, et al: Dose- and schedule-dependent inhibition of the mammalian target of rapamycin pathway with everolimus: a phase I tumor pharmacodynamic study in patients with advanced solid tumors. J Clin Oncol 2008, 26:1603-1610.

22. O'Donnell A, Faivre S, Burris HA, Rea D, Papadimitrakopoulou V, Shand N, Lane HA, Hazell K, Zoellner U, Kovarik JM, et al: Phase I pharmacokinetic and pharmacodynamic study of the oral mammalian target of rapamycin inhibitor everolimus in patients with advanced solid tumors. J Clin Oncol 2008, 26:1588-1595.

23. Duran I, Kortmansky J, Singh D, Hirte H, Kocha W, Goss G, Le L, Oza A Nicklee T, Ho J, et al: A phase II clinical and pharmacodynamic study of temsirolimus in advanced neuroendocrine carcinomas. Br J Cancer 2006, 95:1148-1154

24. Darb-Esfahani S, Faggad A, Noske A, Weichert W, Buckendahl AC, Muller B, Budczies J, Roske A, Dietel M, Denkert C: Phospho-mTOR and phospho$4 \mathrm{EBP} 1$ in endometrial adenocarcinoma: association with stage and grade in vivo and link with response to rapamycin treatment in vitro. $J$ Cancer Res Clin Oncol 2009, 135:933-941.

25. Dilling MB, Germain GS, Dudkin $L$, Jayaraman AL, Zhang $X$, Harwood FC, Houghton PJ: 4E-binding proteins, the suppressors of eukaryotic initiation factor $4 \mathrm{E}$, are down-regulated in cells with acquired or intrinsic resistance to rapamycin. J Biol Chem 2002, 277:13907-13917.

26. Iwenofu OH, Lackman RD, Staddon AP, Goodwin DG, Haupt HM, Brooks JS: Phospho-S6 ribosomal protein: a potential new predictive sarcoma marker for targeted mTOR therapy. Mod Pathol 2008, 21:231-237.

27. Cho D, Signoretti S, Dabora S, Regan M, Seeley A, Mariotti M, Youmans A, Polivy A, Mandato L, McDermott D, et al: Potential histologic and molecular predictors of response to temsirolimus in patients with advanced renal cell carcinoma. Clin Genitourin Cancer 2007, 5:379-385.
28. Hughes TA, Brady HJ: Cross-talk between pRb/E2F and Wnt/beta-catenin pathways: E2F1 induces axin2 leading to repression of Wnt signalling and to increased cell death. Exp Cell Res 2005, 303:32-46.

29. Hughes TA, Brady HJM: Expression of axin2 is regulated by the alternative $5^{\prime}$ untranslated regions of its mRNA. J Biol Chem 2005, 280:8581-8588

30. Pyronnet $\mathrm{S}$, Imataka H, Gingras AC, Fukunaga R, Hunter T, Sonenberg N: Human eukaryotic translation initiation factor $4 \mathrm{G}$ (elF4G) recruits mnk1 to phosphorylate elF4E. EMBO J 1999, 18:270-279.

31. Coleman LJ, Peter MB, Teall TJ, Brannan RA, Hanby AM, Honarpisheh $H$, Shaaban AM, Smith L, Speirs V, Verghese ET, et al: Combined analysis of elF4E and 4E-binding protein expression predicts breast cancer survival and estimates elF4E activity. Br J Cancer 2009, 100:1393-1399.

32. Smith L, Brannan RA, Hanby AM, Shaaban AM, Verghese ET, Peter M, Pollock S, Satheesha S, Szynkiewicz M, Speirs V, Hughes TA: Differential regulation of estrogen receptor $\beta$ isoforms by $5^{\prime}$ untranslated regions in cancer. Journal of Cellular and Molecular Medicine 2010, 14:2172-2184.

33. Smith L, Coleman LJ, Cummings M, Satheesha S, Shaw SO, Speirs V, Hughes TA: Expression of oestrogen receptor beta isoforms is regulated by transcriptional and post-transcriptional mechanisms. Biochem J 2010, 429:283-290

34. Akamine R, Yamamoto $T$, Watanabe $M$, Yamazaki N, Kataoka M, Ishikawa M, Ooie T, Baba Y, Shinohara Y: Usefulness of the $5^{\prime}$ region of the cDNA encoding acidic ribosomal phosphoprotein $\mathrm{PO}$ conserved among rats, mice, and humans as a standard probe for gene expression analysis in different tissues and animal species. J Biochem Biophys Methods 2007, 70:481-486.

35. Livak KJ, Schmittgen TD: Analysis of relative gene expression data using real-time quantitative PCR and the 2(-Delta Delta C(T)) Method. Methods 2001, 25:402-408.

36. Macaskill EJ, Bartlett JM, Sabine VS, Faratian D, Renshaw L, White $S$, Campbell FM, Young O, Williams L, Thomas JS, Barber MD, Dixon JM: The mammalian target of rapamycin inhibitor everolimus (RAD001) in early breast cancer: results of a pre-operative study. Breast Cancer Res Treat.

37. Going JJ: Efficiently estimated histologic cell counts. Hum Pathol 1994, 25:333-336.

38. Hsieh AC, Costa M, Zollo O, Davis C, Feldman ME, Testa JR, Meyuhas O, Shokat KM, Ruggero D: Genetic dissection of the oncogenic mTOR pathway reveals druggable addiction to translational control via 4EBPelF4E. Cancer Cell 2010, 17:249-261.

39. Gingras AC, Raught B, Gygi SP, Niedzwiecka A, Miron M, Burley SK, Polakiewicz RD, Wyslouch-Cieszynska A, Aebersold R, Sonenberg N: Hierarchical phosphorylation of the translation inhibitor 4E-BP1. Genes Dev 2001, 15:2852-2864.

40. Mothe-Satney I, Yang D, Fadden P, Haystead TA, Lawrence JC Jr: Multiple mechanisms control phosphorylation of PHAS-I in five (S/T)P sites that govern translational repression. Mol Cell Biol 2000, 20:3558-3567.

41. Gingras AC, Gygi SP, Raught B, Polakiewicz RD, Abraham RT, Hoekstra MF, Aebersold R, Sonenberg N: Regulation of 4E-BP1 phosphorylation: a novel two-step mechanism. Genes Dev 1999, 13:1422-1437.

42. Noh WC, Mondesire WH, Peng J, Jian W, Zhang H, Dong J, Mills GB, Hung MC, Meric-Bernstam F: Determinants of rapamycin sensitivity in breast cancer cells. Clin Cancer Res 2004, 10:1013-1023.

43. Chang SB, Miron P, Miron A, Iglehart JD: Rapamycin inhibits proliferation of estrogen-receptor-positive breast cancer cells. J Surg Res 2007, 138:37-44.

44. Hosoi H, Dilling MB, Liu LN, Danks MK, Shikata T, Sekulic A, Abraham RT, Lawrence JC Jr, Houghton PJ: Studies on the mechanism of resistance to rapamycin in human cancer cells. Mol Pharmacol 1998, 54:815-824.

45. Wu GJ, Sinclair CS, Paape J, Ingle JN, Roche PC, James CD, Couch FJ: 17q23 amplifications in breast cancer involve the PAT1, RAD51C, PS6K, and SIGma1B genes. Cancer Res 2000, 60:5371-5375.

46. Jastrzebski K, Hannan KM, Tchoubrieva EB, Hannan RD, Pearson RB: Coordinate regulation of ribosome biogenesis and function by the ribosomal protein $\mathrm{S} 6$ kinase, a key mediator of mTOR function. Growth Factors 2007, 25:209-226.

doi:10.1186/1476-4598-10-19

Cite this article as: Satheesha et al:: Response to mTOR inhibition: activity of eIF4E predicts sensitivity in cell lines and acquired changes in elF4E regulation in breast cancer. Molecular Cancer 2011 10:19. 